\title{
A Multi-Mode Broadband Vibration Energy Harvester Based on MEMS 3D Coils
}

\author{
Hanxiao WU ${ }^{\mathrm{a}, \mathrm{b}, \mathrm{c}}$, Zhi TAO ${ }^{\mathrm{a}, \mathrm{d}}$, Haiwang $\mathrm{LI}^{\mathrm{a}, \mathrm{d}}$, Tiantong $\mathrm{XU}^{\mathrm{a}, \mathrm{d}, 1}$, Wenbin WANG ${ }^{\mathrm{a}, \mathrm{b}}$ and \\ Jiamian SUN ${ }^{\mathrm{a}, \mathrm{b}}$ \\ a National Key Laboratory of Science and Technology on Aero-Engine Aero- \\ thermodynamics, Beihang University, Beijing 100191, China \\ ${ }^{\mathrm{b}}$ School of Energy and Power Engineering, Beihang University, Beijing 100191, China \\ ${ }^{\mathrm{c}}$ ShenYuan Honors College of Beihang University, Beihang University, Beijing 100191, \\ China \\ ${ }^{\mathrm{d}}$ Research Institute of Aero-Engine, Beihang University, Beijing 100191, China
}

\begin{abstract}
This paper presents an electromagnetic vibration energy harvester utilizing 3D MEMS coils and multi-mode structure to improve the output power and broaden the frequency band. We fabricated and assembled the prototype, with a pair of 3D coils fabricated by lithography, silicon etching, silicon direct bonding and copper electroplating, et al., which are compatible with CMOS processes. The numerical simulation was conducted to analysis the vibration modes of the springmass system, which revealed the multi-mode mechanism of serpentine springs. We also tested the output power-frequency curves for different load and excitation acceleration to investigate the optimal load resistance and the influence of excitation. The test results showed that the proposed prototype can generate $1.2 \mu \mathrm{W}$ power under $992 \mathrm{~Hz}$ for $1 \mathrm{~g}$ acceleration with a half-power bandwidth of $65 \mathrm{~Hz}$, which are higher than some recent published data, proving the superiority of proposed structure.
\end{abstract}

Keywords. Vibration energy harvester, MEMS, 3D coils, broadband

\section{Introduction}

With the demand for sustainable and renewable energy increases, it is useful to develop alternative energy strategies, such as harvesting vibration energy. And vibration energy harvesting is a potential technology to replace batteries for wireless sensor networks. Therefore, the vibration energy harvesting technology has attracted the attention of many researches. The mechanisms utilized for vibration energy harvesters (VEH) include electromagnetic, piezoelectric, electrostatic and triboelectric, among which the electromagnetic vibration energy harvesters (EM-VEHs) have been the primary focus of research due to high power-density and long lifetime.

However, most MEMS EM-VEHs employ planar coils, which cause large magnetic leakage and limit the output performance [1,2]. The MEMS 3D solenoid coil demonstrated by Xu et al. based on high-aspect-ratio through silicon vies (TSV) shows potential to overcome the above-mentioned problems [3, 4]. In Ref. [5], we proposed a

\footnotetext{
${ }^{1}$ Corresponding Author, Tiantong XU, National Key Laboratory of Science and Technology on AeroEngine Aero-thermodynamics, Beihang University, Beijing 100191, China; Email: xutiantong@buaa.edu.cn.
} 
structure scheme design and a dynamic model based on MEMS 3D coils. The application of 3D coils can significantly improve the output power of MEMS VEHs.

Simultaneously, a large number of researches have focused on widening the frequency bandwidth, such as resonance frequency tuning [6], nonlinear dynamics [7, $8]$, multi-mode vibration $[9,10]$. Commonly, the multi-mode vibration is realized by introducing multiple vibrators to form a multi-degree of freedom system. However, the multiple modes are relatively independent, and the resonance frequency bands are far apart, which makes it difficult to match the VEH's resonance frequencies with the environment vibration.

In this paper, we proposed an EM-VEH using 3D MEMS coils. A silicon steel sheet was inserted into the coil to constrain the magnetic field, and hence, to enhancement the output power and efficiency. We also adopted a random design method by utilizing eight planer serpentine springs with high-sensitivity. Under the influence of random disturbance and process deviation, the resonance points of these planer springs will be randomly distributed in a certain frequency band, and consequently, to realize a concentrated multi-mode vibration and broaden the effective working frequency band of EM-VEHs.

\section{Materials \& Methods}

\subsection{Structure Design}

The structure of EM-VEH using MEMS 3D coils is shown as figure 1a. The MEMS coils are embedded in a silicon substrate via deep Si etching, silicon bonding and $\mathrm{Cu}$ electroplating. Each coil has 53 turns. And the height is $2 \mathrm{~mm}$, which is equal to the thickness of two bonded wafers. The wire diameter is $100 \mu \mathrm{m}$, and the turn pitch is also $100 \mu \mathrm{m}$. A silicon steel sheet is inserted to the coils to build a closed magnetic loop. The magnet is arranged on the centre plane of two coils and vibrates along the direction perpendicular to the coil axis. The arrangement of coils and silicon steel sheet is shown as figure $1 \mathrm{~b}$. A pair of planar copper springs are glued on both side of the bonded substrate. The structure parameters of springs are shown in figure $1 \mathrm{c}$.

Under the excitation of external environment, the relative position between the permanent magnet and the silicon steel sheet changes, thereby generating an induced electromotive force in the coils. The application of 3D coils and silicon steel sheet can increase the flux gradient during vibrating, and consequently, improve the output power.

These two planar springs total have eight long serpentine springs with thickness of $0.2 \mathrm{~mm}$. This structure has a lower stiffness coefficient along vibration direction, and a larger stiffness coefficient along non-vibration direction. And these springs have a large number of turns, which makes the resonance modes instable to a certain extent. With the deviation of processing and assembly, the vibration modes of different springs are easily to separate from each other, thereby broadening the resonance frequency band. 
(a)

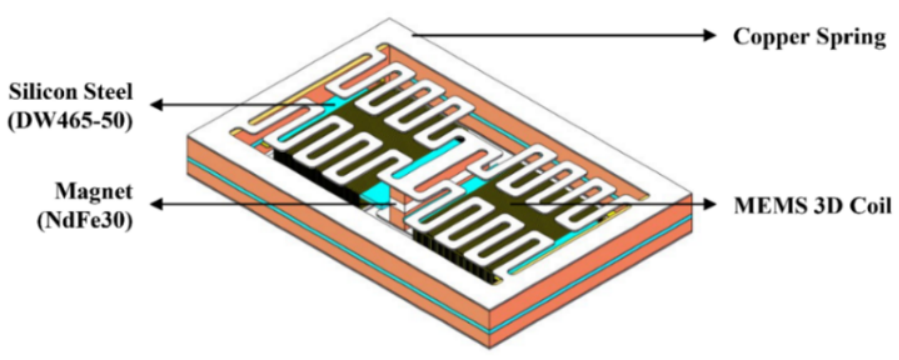

(b)

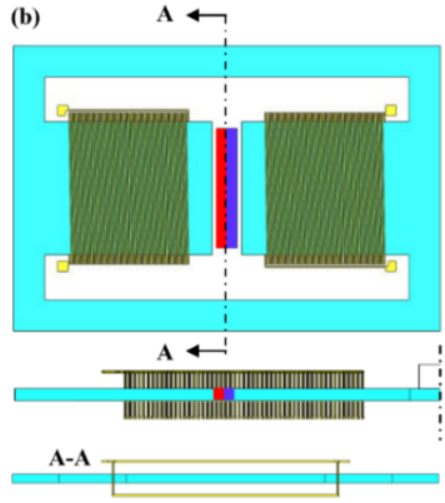

(c)

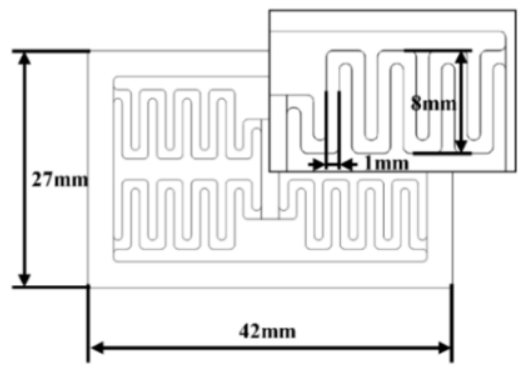

Figure 1. (a) The overall structure design of VEH; (b) the arrangement of coils and iron core; (c) structure of planar spring.

\subsection{Prototype Fabrication}

The coils of this device are fabricated by MEMS technology. Figure 2 shows the fabrication process of $3 \mathrm{D}$ coils. The whole process includes two stages. The first stage includes silicon etching and silicon bonding, corresponding to step (1-9). The substrates used are $1 \mathrm{~mm}$-thick double-polished intrinsic silicon wafer. A silicon mould with through holes, horizontal wire grooves and a silicon steel sheet groove is fabricated by silicon oxide etching and double-side silicon deep etching process. Then two layers of silicon wafers are aligned and bonded to form a complete $3 \mathrm{D}$ loops and a silicon steel sheet space inside the substrate.

The second stage is to fill the copper into the silicon mould by electroplating, corresponding to step (10-12). Firstly, a seed layer is sputtered on the back side of the bonded substrate. And then, electroplate $\mathrm{Cu}$ until it fills the horizontal grooves and the through holes. Finally, remove the excess $\mathrm{Cu}$ on the surface by chemical mechanical polishing. 


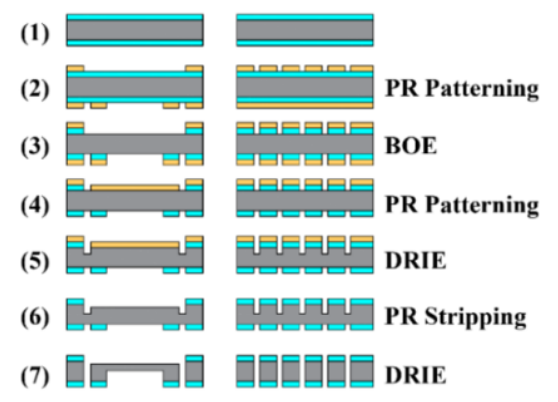

(8)

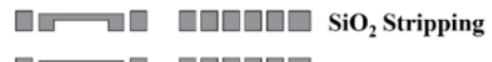

(9)
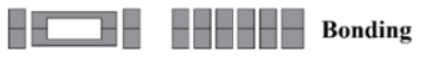

(10)
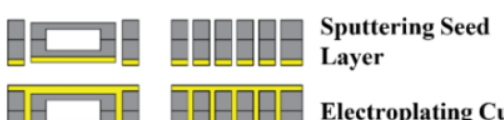

(11)
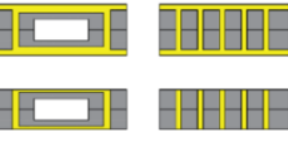

Electroplating $\mathrm{Cu}$

(12)

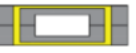

Chemical Mechanical Polishing

Silicon Substrate

$\mathrm{SiO}_{2}$

Photoresist

$\mathrm{Cu}$

Figure 2. Fabrication process of MEMS 3D coils.

Figure 3 exhibits the coil samples during the fabrication. And we tested the electrical performance of the coils. The total size of single coil is $13.1 \mathrm{~mm} \times 17.4 \mathrm{~mm}$. The tested coil resistance is $3.91 \Omega$, and the DC inductance after inserting the iron core is $249.06 \mu \mathrm{H}$.

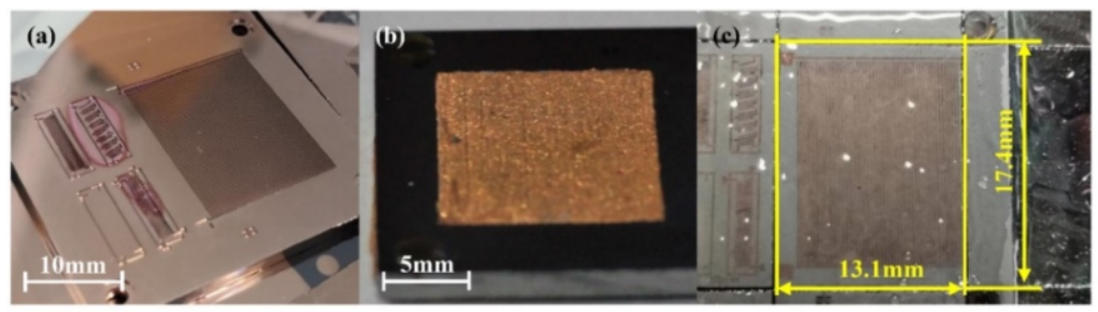

Figure 3. Coil samples after (a) silicon direct bonding, (b) electroplating, and (c)CMP and dicing.

Figure 4a illustrates the assembly process of the prototype. Firstly, align and fix the bottom spring and permanent magnet. Then insert the silicon steel sheets into the coils and fix them on both sides of the permanent magnet, while ensuring a suitable air gap size, which is $0.4 \mathrm{~mm}$ in this study. In this step, it is also necessary to connect the two silicon steel sheets with magnetic conductive glue to realize the closure of the magnetic circuit. Next, connect the signal wire to the electrodes of the coils. Finally, install the upper spring to complete the assembly of the prototype. Figure $4 \mathrm{~b}$ is the prototype photo after the assembly process. In this design, we also added some auxiliary structures to limit the vibration amplitude and improve the reliability.

(a) (1)
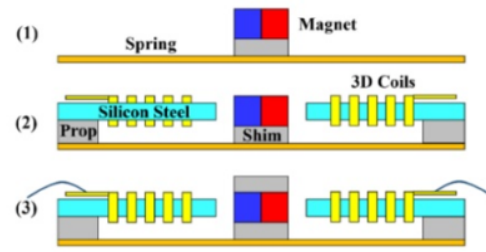

(4)

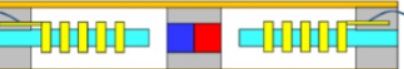

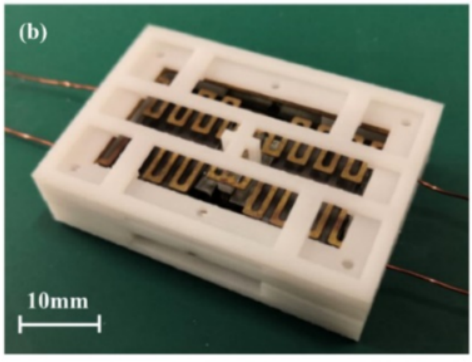

Figure 4. (a) Assembly process of prototype, and (b) the prototype photo. 


\subsection{Modal Analysis}

In this study, we conducted a simulation analysis on the vibration modes of the mechanical system composed of the vibrator and springs by using ANSYS software, mainly focusing on the modal changes of the springs under different frequency excitations. Figure 5a shows the simulating model.
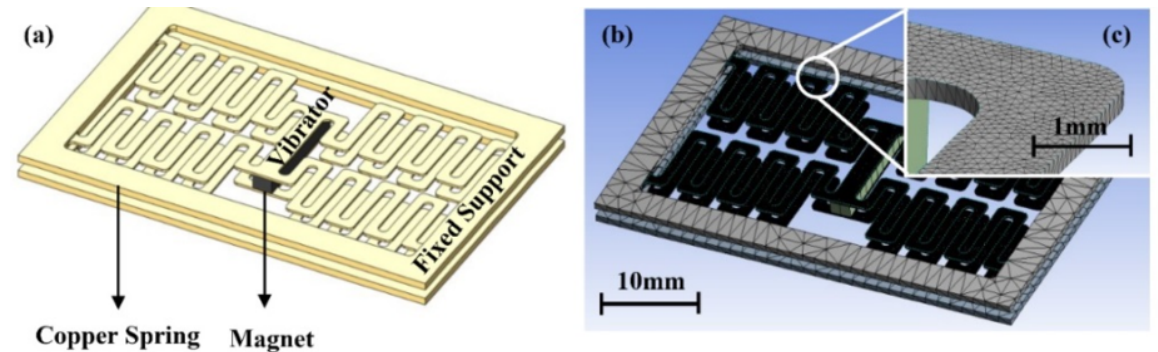

Figure 5. (a) Simulation model and (b) computational grid.

The computational grid is demonstrated in figure $5 \mathrm{~b}$. The number of elements is 589,988 , and the nodes number is $1,090,035$. In this simulation, we did not consider the electromagnetic effects of iron core and coils. Hence, in the calculation results, the modes which should be associated with the electromagnetic effect have no practical reference significance. In consequence, we only analysis the modal bifurcation phenomenon of the springs. The relevant modes and corresponding frequencies of each order are listed in figures 6a-6e. It can be seen that the springs have multiple vibration modes around $1146 \mathrm{~Hz}$, indicating that the modes of springs are quite unstable and easily disturbed by random factors. Therefore, it can also be judged that due to the inevitable random disturbances such as processing and assembly deviation, the actual vibration modes of springs will diverge near the resonance point, resulting in a broadband response.

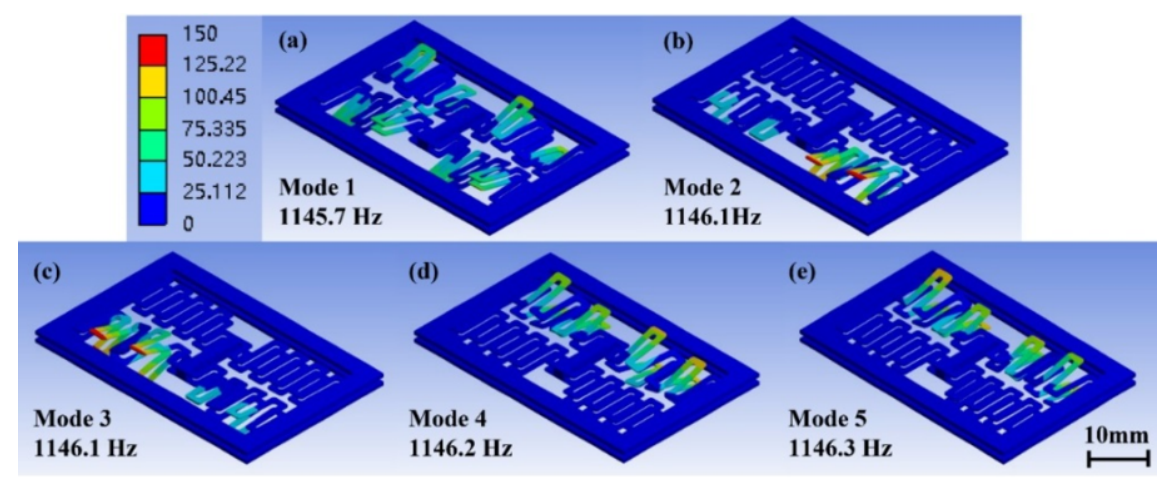

Figure 6. Vibration modes for spring-mass system. 


\subsection{Experiment Apparatus}

We connected an adjustable resistor with one of the coils to form a closed load circuit, shown as figures $7 \mathrm{a}$ and $7 \mathrm{~b}$. By testing the voltage of the load resistance, the output power of the VEH can be converted. Since the two coils are completely equivalent, in this study, we tested the output power of one coil in this device to simplify the experiment process.

The test system is illustrated in figure 7c. The VEH is excited by a portable shaker (PCB 9100D), which integrates a closed-loop control system to generate the vibration excitation of given amplitude and frequency. The voltage signal is collected by a data acquisition card (DAQ, Advantech USB4713) and then processed by LabVIEW platform.

(a)

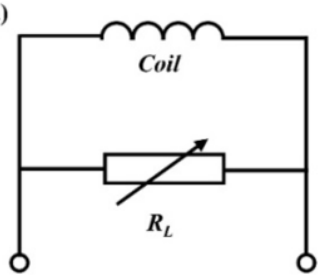

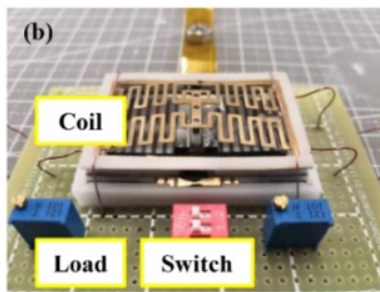

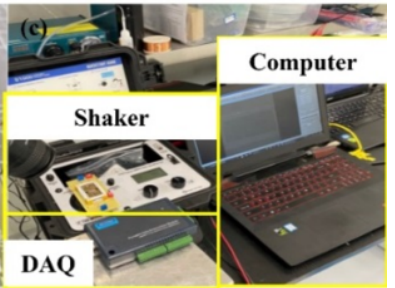

Figure 7. (a) The experiment circuit, (b) photo of circuit and (c) experiment system.

During testing, the excitation frequency was increased or decreased gradually while the amplitude was kept constant. We tested the steady-state signal of output voltage at each excitation frequency. Then the output power at each frequency can be calculated by using the root mean square (RMS) voltage.

\section{Results}

\subsection{Influence of Load Resistance}

First, the output performance under up-sweep and down-sweep frequency are compared under $1 \mathrm{~g}\left(1 \mathrm{~g}=9.8 \mathrm{~m} / \mathrm{s}^{2}\right)$ acceleration and $10 \Omega$ load. Figure 8 shows the output power changes with frequency of excitation. It can be seen that the output power for up-sweep and down-sweep is almost equal. Therefore, in subsequent experiments, we only tested the output power during up-sweep process. In addition, the VEH also exhibits multi-peak characteristic in tested frequency band $(850-1100 \mathrm{~Hz})$, which is similar to the simulation results, indication that there is a bifurcation phenomenon of vibration modes in this frequency band. Compared with single frequency vibration, the output bandwidth has been broadened. 


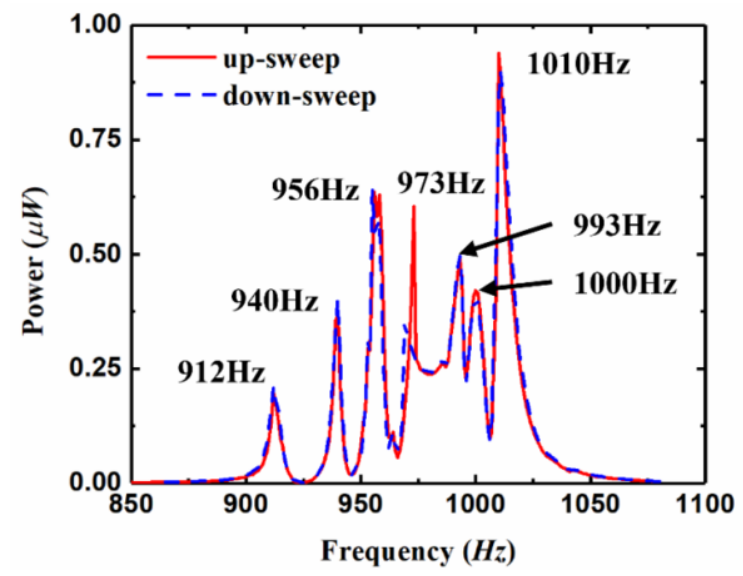

Figure 8. The power-frequency curves for up-sweep and down-sweep.

Then we test the output power for different load resistance from $2.5 \Omega$ to $15.0 \Omega$ under $1 \mathrm{~g}$ acceleration and frequency band form $850 \mathrm{~Hz}$ to $1100 \mathrm{~Hz}$ to determine the optimal load for this device. Figures 9a-9e shows the power-frequency curve for $2.54 \Omega$, $3.74 \Omega, 5.04 \Omega, 10.0 \Omega$ and $15.0 \Omega$ separately. The amplitude-frequency response of the VEH remains stable and repeatable under different load resistances. The peak power at resonance frequency for different load resistance are depicted in figure 9f, indicating that the optimal load resistance appears at $3.74 \Omega$, which is approximately equal to the coil resistance. The maximum output power reaches at $1.20 \mu \mathrm{W}$ for $1 \mathrm{~g}$ excitation acceleration, meanwhile relatively high output power appears in frequency band of $910-916 \mathrm{~Hz}, 937-942 \mathrm{~Hz}, 953-960 \mathrm{~Hz}, 971-1002 \mathrm{~Hz}$ and $1008-1013 \mathrm{~Hz}$, with a total halfpower bandwidth of $54 \mathrm{~Hz}$.
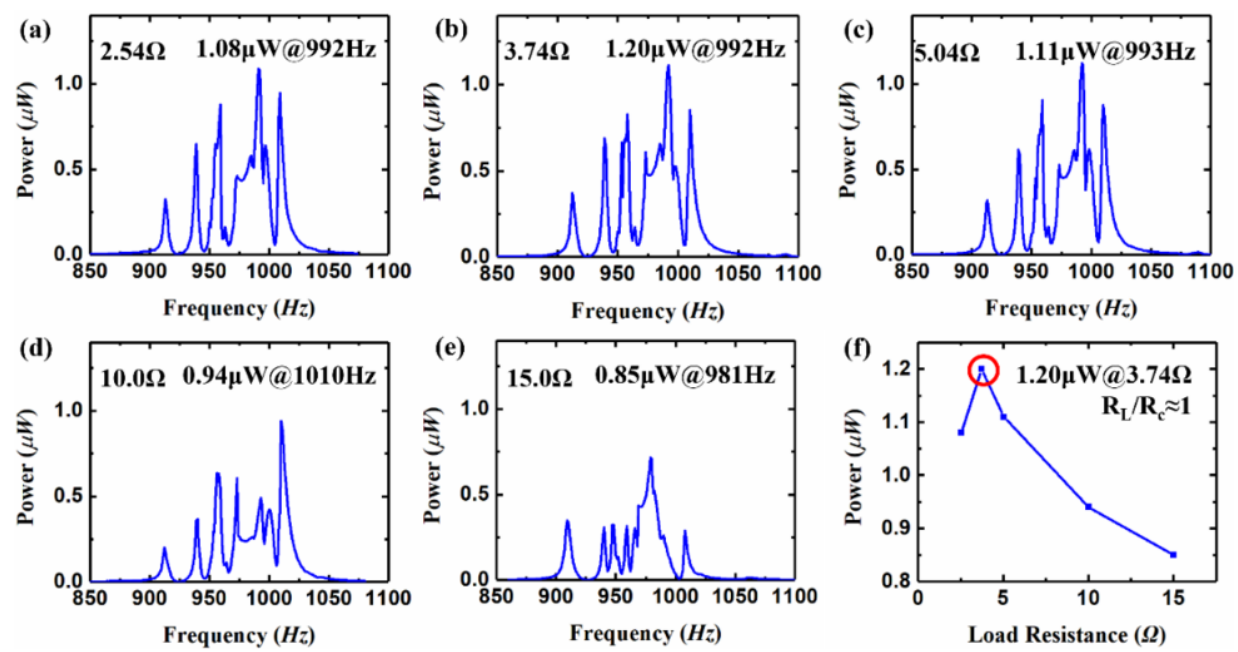

Figure 9. (a)-(e) The output power-frequency curves for different load resistances and (f) the relationship between peak power and load resistance. 


\subsection{Influence of Excitation Acceleration}

We also investigated the influence of excitation acceleration on the output performance of $\mathrm{VEH}$, shown as figures $10 \mathrm{a}-10 \mathrm{~b}$, which illustrates the power-frequency curves for load resistance of $2.54 \Omega$ and $3.74 \Omega$ separately. Compared with the output performance under $1 \mathrm{~g}$ excitation, the output power of VEH under $2 \mathrm{~g}$ excitation has been significantly improved. Simultaneously, the amplitude-frequency cure still exhibits multi-peak characteristics, and the resonance peak frequency is almost unchanged. The difference is that the maximum power appears at $981 \mathrm{~Hz}$ and $982 \mathrm{~Hz}$ for $2.54 \Omega$ and $3.74 \Omega$ separately, while it is $992 \mathrm{~Hz}$ for both load resistances under $1 \mathrm{~g}$ excitation. In addition, the excitation acceleration does not influence the optimal load resistance, which is also $3.74 \Omega$ for $2 \mathrm{~g}$ excitation.

However, with the increase of input acceleration, the normalized power density $\left(\mathrm{NPD}, \mathrm{NPD}=\right.$ output power/(volume $\left.\left.\times \mathrm{a}^{2}\right)\right)$ decreases. For $1 \mathrm{~g}$ acceleration, the normalized power density is $0.16 \mu \mathrm{W} / \mathrm{cm}^{3} \mathrm{~g}^{2}$, while for $2 \mathrm{~g}$ acceleration, this value is $0.12 \mu \mathrm{W} / \mathrm{cm}^{3} \mathrm{~g}^{2}$.

Additionally, the output power has relatively high value in frequency band of 936$942 \mathrm{~Hz}, 949-953 \mathrm{~Hz}, 957-962 \mathrm{~Hz}, 973-996 \mathrm{~Hz}$ and $1009-1015 \mathrm{~Hz}$, with a total half-power bandwidth of $44 \mathrm{~Hz}$, which is slightly narrower than that of $1 \mathrm{~g}$ acceleration.
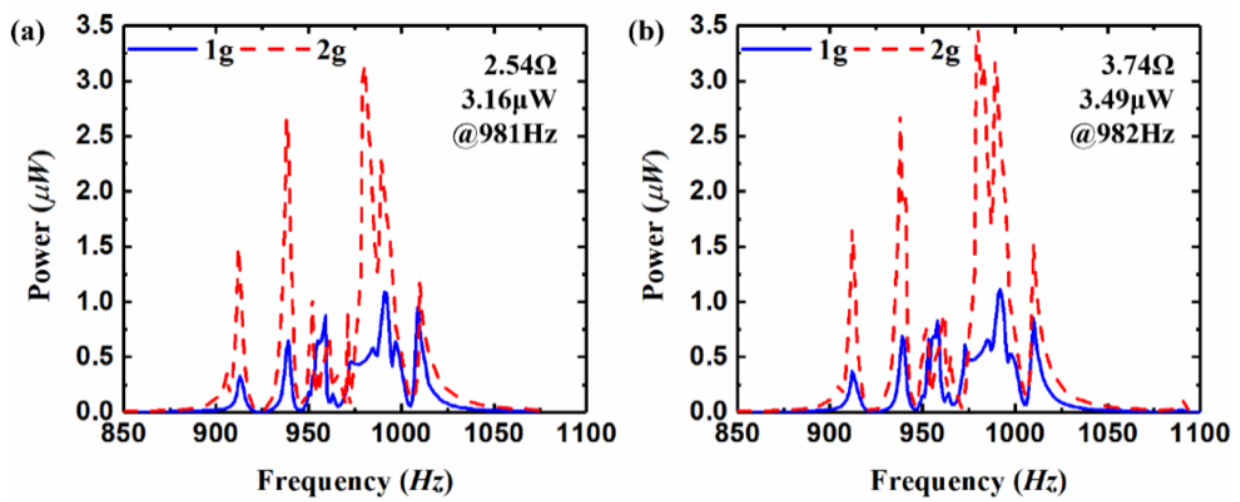

Figure 10. The output power-frequency curves under $1 \mathrm{~g}$ and $2 \mathrm{~g}$ acceleration for (a) $2.54 \Omega$ load and (b) $3.74 \Omega$ load.

Table 1 summarized the output performance of several recently published researches about broadband vibration energy harvester. It can be seen that the proposed structure in this paper has higher output power and broader frequency bandwidth than that of some previously published data on VEHs, which also proves that the structure utilizing $3 \mathrm{D}$ coils and broadband design method proposed in this paper are advantageous.

Table 1. Summary of the reviewed VEHs output performance.

\begin{tabular}{lllll}
\hline Ref. & Output power $(\boldsymbol{\mu W})$ & Acceleration $(\mathbf{g})$ & $\mathbf{N P D}\left(\boldsymbol{\mu W} / \mathbf{c m}^{\mathbf{3}} \mathbf{g}^{\mathbf{2}}\right)$ & Frequency bandwidth $(\mathbf{H z})$ \\
\hline$[11]$ & $5 \times 10^{-4}$ & 1 & $1.56 \times 10^{-2}$ & 19 \\
{$[12]$} & $5.5 \times 10^{-2}$ & 1 & 0.157 & - \\
{$[13]$} & 2 & 7.5 & 0.0432 & 40 \\
{$[14]$} & $2.3 \times 10^{-3}$ & 1.5 & $1.07 \times 10^{-3}$ & 46 \\
Proposed & 1.2 & 1 & 0.16 & 54 \\
\hline
\end{tabular}




\section{Conclusion}

This paper presents an EM-VEH based on CMOS-compatible 3D MEMS coils and multi-mode structure. A pair of silicon steel sheets are inserted into the coils to close the magnetic path. Two planar springs which including eight serpentine branches are installed on the top and bottom surface of the VEH.

MEMS technologies including lithography, deep silicon etching and TSV electroplating are applied to fabricate the 3D coils. Consequently, the VEH prototype is assembled by integrating the iron cores, springs, magnet and signal wires. Numerical simulation is conducted to analysis the vibration mode of the spring-mass system. The results indicate that the vibration modes of serpentine springs are unstable and will separate from each other under random disturbance, which has the potential to broaden the frequency band of VEH.

Additionally, we test the output performance of the VEH prototype in $850-1100 \mathrm{~Hz}$ frequency range. The output power reaches maximum value when load resistance is $3.74 \Omega$, which is approximately equal to the coil resistance. Under $1 \mathrm{~g}$ acceleration, the peak power is $1.2 \mu \mathrm{W}$ with half-power bandwidth of $54 \mathrm{~Hz}$, corresponding to the NPD of $0.16 \mu \mathrm{W} / \mathrm{cm}^{3} \mathrm{~g}^{2}$. When the excitation acceleration increases to $2 \mathrm{~g}$, the peak output power also raises to $3.49 \mu \mathrm{W}$, while the NPD reduces slightly to $0.12 \mu \mathrm{W} / \mathrm{cm}^{3} \mathrm{~g}^{2}$. The peak power and half-power bandwidth of the VEH proposed in this paper are improved compared with the output performance of some published data.

\section{Acknowledgement}

This work was funded by the High-speed moving component dynamic tester (2017YFF0107601 and 2017YFF0107604), the Beijing Municipal Natural Science Foundation (No. JQ20016), Beijing Municipal Science and Technology Project (No. Z201100007720006) and the National Nature Science Foundation of China (No. 52006003).

\section{References}

[1] Williams C B, Shearwood C, Harradine M A, Mellor P H, Birch T S and Yates R B 2001 Development of an electromagnetic micro-generator IEE Proceedings-Circuits Devices Syst. 148 (6) 337-42.

[2] Wang K, Dai X, Xiang X, Ding G and Zhao X 2019 A MEMS-based bi-stable electromagnetic energy harvester with an integrated magnetization-reversible circuit 2019 20th International Conference on Solid-State Sensors, Actuators and Microsystems \& Eurosensors XXXIII (TRANSDUCERS \& EUROSENSORS XXXIII) pp 358-61.

[3] Li H, Liu J, Xu T, Xia J, Tan X and Tao Z 2018 Fabrication and optimization of high aspect ratio through-silicon-vias electroplating for 3D inductor Micromachines 9 (10) 528.

[4] Xu T, Sun J, Wu H, Li H, Li H and Tao Z 2019 3D MEMS in-chip solenoid inductor with high inductance density for power MEMS device IEEE Electron Device Lett. 40 (11) 1816-9.

[5] Tao Z, Wu H, Li H, Li H, Xu T, Sun J and Wang W 2020 Theoretical model and analysis of an electromagnetic vibration energy harvester with nonlinear damping and stiffness based on 3D MEMS coils J. Phys. D. Appl. Phys. 53495503.

[6] Sari I, Balkan T and Külah H 2010 An electromagnetic micro power generator for low-frequency environmental vibrations based on the frequency up conversion technique J. Microelectromechanical Syst. 19 (1) 14-27.

[7] Huang D, Zhou S and Litak G 2019 Theoretical analysis of multi-stable energy harvesters with highorder stiffness terms Commun. Nonlinear Sci. Numer. Simul. 69 270-86. 
[8] Barton D A W, Burrow S G and Clare L R 2010 Energy harvesting from vibrations with a nonlinear oscillator J. Vib. Acoust. Trans. ASME 132 (2) 0210091-7.

[9] Yang B, Lee C, Xiang W, Xie J, Han He J, Kotlanka R K, Low S P and Feng H 2009 Electromagnetic energy harvesting from vibrations of multiple frequencies J. Micromechanics Microengineering 19 (3) 035001.1-8

[10] Tao K, Wu J, Tang L, Xia X, Lye S W, Miao J and Hu X 2016 A novel two-degree-of-freedom MEMS electromagnetic vibration energy harvester J. Micromechanics Microengineering 26 (3) 35020.

[11] Liu H, How Koh K and Lee C 2014 Ultra-wide frequency broadening mechanism for micro-scale electromagnetic energy harvester Appl. Phys. Lett. 104 (5) 053901.

[12] Liu H, Qian Y and Lee C 2013 A multi-frequency vibration-based MEMS electromagnetic energy harvesting device Sensors Actuators: A Phys. 204 37-43.

[13] Zhang Q, Kim E S. 2016 Microfabricated Electromagnetic Energy Harvesters with Magnet and Coil Arrays Suspended by Silicon Springs IEEE Sens. J. 16 (3) 634-41.

[14] Wang Y, Zhang Q, Zhao L and Kim E S 2015 Ferrofluid liquid spring for vibration energy harvesting Proc. IEEE Int. Conf. Micro Electro Mech. Syst. Feb 2015 pp 122-5. 